\title{
Construção de uma escala de mensuração de avaliação no campo de políticas públicas de computação em nuvem
}

Marcos Fernando Machado de Medeiros

Doutor em Administração pelo PPGA/UFRN. Professor Adjunto do Departamento de Ciências Administrativas na Universidade Federal do Rio Grande do Norte (UFRN)

mfmedeiros@gmail.com

Lucas dos Santos Costa

Mestrando em Administração pelo Programa de Pós-Graduação em Administração da Faculdade de Economia, Administração e Contabilidade da Universidade de São Paulo (FEA-USP).

adm.lucassantos@hotmail.com

Igor Vinicius de Lucena Diniz

Graduando em Administração pela Universidade Federal do Rio Grande do Norte (UFRN).

igor.diniz72@gmail.com

\section{Editor Científico: José Edson Lara}

Organização Comitê Científico

Double Blind Review pelo SEER/OJS

2Aprovado em 06.11.2018

\section{(c) (i) (8)}

Este trabalho foi licenciado com uma Licença Creative Commons - Atribuição - Não Comercial 3.0 Brasil 


\title{
Resumo
}

O objetivo deste trabalho é mensurar a confiabilidade de um instrumento para avaliação das políticas públicas de computação em nuvem no Poder Legislativo de um estado brasileiro. Foi realizada uma pesquisa com gestores da área de Tecnologia da Informação, por meio da aplicação de questionários fechados, com a utilização de escala de tipo Likert. A análise consiste na mensuração de confiabilidade de coerência interna mediante Coeficiente de Alfa de Cronbach. Os resultados foram analisados individualmente em suas categorias: capacitação, implantação, infraestrutura, modelo, organizacional, políticas públicas e recursos. Como pesquisa de caráter exploratório, obteve resultado a níveis aceitáveis pela literatura apenas na confiabilidade individual de dois construtos, apesar do instrumento ter sido classificado como confiável ao considerar todas as variáveis. A partir dos resultados da pesquisa, o modelo poderá ser testado em outras realidades e após esse primeiro passo as categorias poderão vir a se tornar instrumentos de avaliação independentes.

Palavras-Chave: Administração Pública; Políticas Públicas; Computação em Nuvem; Confiabilidade.

\section{Construction of an evaluation measurement scale in the field of cloud computing public policies}

\begin{abstract}
The objective of this papers is to measure the reliability of an instrument for the evaluation of the public policies of cloud computing in the Legislative Branch of a brazilian state. A survey was conducted with CIO's through the application of closed questionnaires using Likert scale. The analysis consists of the measurement of internal consistency reliability using Cronbach's Alpha Coefficient. The results were analyzed individually in their categories: capacity building, implementation, infrastructure, model, organizational, public policies and resources. As an exploratory research, results show acceptable levels in the literature only in the individual reliability of two constructs, although the instrument was classified as reliable when considering all variables. From the results of the research, the model can be tested in other realities and after this first step categories may become independent evaluation instruments.
\end{abstract}

Keywords: Public Administration; Public Policies; Cloud Computing; Reliability.

\section{Construcción de una escala de medición de evaluación en el ámbito de las políticas públicas de computación en nube}

\section{Resumen}

El objetivo de este trabajo es medir la confiabilidad de un instrumento para la evaluación de las políticas públicas de computación en nube en el Poder Legislativo de un estado brasileño. Se realizó una investigación con gestores del área de Tecnología de la Información, mediante aplicación de cuestionarios cerrados, con la utilización de escala de tipo Likert. El análisis consiste en la medición de confiabilidad de coherencia interna por intermedio del Coeficiente 
de Alfa de Cronbach. Los resultados fueron analizados individualmente en sus categorías: capacitación, implantación, infraestructura, modelo, organización, políticas públicas y recursos. Como investigación de carácter exploratorio, obtuvo resultados a niveles aceptables por la literatura sólo en la confiabilidad individual de dos constructos, a pesar de que el instrumento fue clasificado como confiable al considerar todas las variables. A partir de los resultados de la investigación, el modelo puede ser probado en otras realidades y después de ese primer paso las categorías podrían convertirse en instrumentos de evaluación independientes.

Palabras-clave: Administración Pública; Políticas públicas; Computación en Nube; Fiabilidad.

\section{Introdução}

Computação em nuvem é considerada por diversos autores como um novo paradigma dentre as Tecnologias da Informação (TI), visto que provoca modificações estruturais nos departamentos de TI, pela transformação das suas atividades em serviços. É o conceito de XaaS - tudo como serviço (Xu, 2012) ou TIaaS - TI como serviço (Veras, 2012). No tocante a este tema, o estudo de Medeiros (2014) identificou alguns benefícios da computação em nuvem para o serviço público, entre eles redução de custos, agilidade e melhoria dos serviços que se alinham com a necessidade de aumentar a eficiência no serviço público.

Além dos benefícios, existem limitações ao seu uso, desafios, ou ainda riscos presentes na literatura sobre computação em nuvem. Dentre eles destaca-se a segurança da informação, disponibilidade e a dependência da Internet (Medeiros, 2014), que tem forte influência sobre os serviços de computação em nuvem. Em um país como o Brasil, de grande extensão territorial, onde a Internet não alcança algumas regiões com a qualidade necessária, a exemplo das áreas rurais ou distantes dos grandes centros e capitais dos estados, os serviços de computação em nuvem podem ficar comprometidos.

Nos estudos em organizações públicas (Medeiros, 2014; Medeiros \& Sousa Neto, 2014, 2016) se observou como ocorre a inserção desta tecnologia no ambiente governamental, desde a concepção das políticas públicas até o seu efetivo uso. Estudos já foram realizados no Poder Executivo e no Poder Judiciário, no entanto, ainda não se evidenciam estudos que busquem compreender como o Poder Legislativo se posiciona em relação ao uso da computação em nuvem.

Portanto, esta pesquisa tem por objetivo principal mensurar a confiabilidade de um instrumento para avaliação das políticas públicas de computação em nuvem no Poder Legislativo de um estado brasileiro, no que se refere à sua forma de utilização, como na efetivação da computação em nuvem como uma política pública de tecnologia da informação. 
O artigo tem como objetivo secundário comparar a mensuração inicial da confiabilidade, presença de todas as variáveis, com os resultados otimizados das categorias, retirada de variáveis menos/não confiáveis.

\section{Referencial teórico}

Na primeira parte, o referencial teórico conta as principais conceituações de Computação em Nuvem, logo em seguida com o destaque das vantagens e desvantagens, e por fim, com a políticas públicas de computação em nuvem.

\subsection{Conceitos de computação em nuvem (cn)}

O termo computação em nuvem (cloud computing), embora já tratado nos estudos de Carr (2005), e discutido no mercado desde meados de 2006 (Chaves, 2011), ainda suscita algumas discussões no meio acadêmico. Assim, este referencial aborda os conceitos de computação em nuvem, suas características, benefícios e limitações.

Santos, Amelotti e Villar (2012) afirmam que “computação em nuvem está se tornando um componente essencial para novos negócios e organizações já estabelecidas desenvolverem estratégias que envolvam recursos de TI".

O National Institute of Standards and Tecnology [NIST] (2011), afirma que a computação em nuvem pode ser definida como:

um modelo que possibilita acesso, de modo conveniente e sob demanda, a um conjunto de recursos computacionais configuráveis (por exemplo, redes, servidores, armazenamento, aplicações e serviços) que podem ser rapidamente adquiridos e liberados com mínimo esforço gerencial ou interação com o provedor de serviços.

A computação em nuvem se popularizou nos últimos anos, resultado dos constantes avanços tecnológicos, como a capacidade de processamento de dados e a popularização do uso da Internet. Mas ainda existem dúvidas sobre o conceito que melhor define o serviço, já que muitos aspectos técnicos são levados em consideração ao definir a $\mathrm{CN}$. A computação em nuvem representa uma convergência de duas grandes tendências em Tecnologia da Informação: eficiência e agilidade dos negócios (Marston, Li, Bandyopadhyay, Zhang, \& Ghalsasi, 2010). Já Armbrust et al. (2010) afirmam que a computação em nuvem se refere tanto aos aplicativos entregues como serviços através da internet, quanto de hardware e softwares nos datacenters que oferecem esses serviços. Para Tigre e Noronha (2013), 
a computação em nuvem simboliza a tendência de colocar toda a infraestrutura e informação disponível de forma digital na Internet, incluindo os softwares, ferramentas de busca, redes de comunicação, provedores, centros de armazenamento e processamento de dados.

Em paralelo, Vaquero, Rodero-Merino, Caceres e Lindner (2009), estabelecem que a nuvem pode ser entendida como um grande conjunto de recursos virtualizados, de fácil acesso e uso e que os recursos podem ser dinamicamente reconfigurados e ajustados para uma escala variável de acordo com a sua utilização. Em se tratando de definições, o principal modelo conceitual, que é utilizado como referência no mundo todo, é o documento técnico produzido pelo National Institute of Standards and Technology (NIST), intitulado de 'The NIST definition of Cloud Computing', apresentando detalhes sobre a computação em nuvem, como as características essenciais, modelos de serviços e os modelos de implantação.

Ainda de acordo com o NIST (2011), o modelo de computação em nuvem é composto por cinco características essenciais, três modelos de serviço e quatro modelos de implantação. As características citadas são: autoatendimento sob demanda, amplo acesso a serviços de rede, pool de recursos, elasticidade rápida e serviços mensuráveis. A seguir, veremos as definições para cada característica:

- Autoatendimento sob demanda: o usuário pode adquirir recursos computacionais na medida em que necessite e, ainda, sem a necessidade do contato direto com o provedor do serviço;

- Amplo acesso a serviços de rede: os recursos devem estar disponíveis através da rede e devem ser acessíveis por mecanismos padrão, permitindo seu uso por diferentes dispositivos (computadores pessoais, smartphones, tablets, etc.);

- Pool de recursos: os recursos computacionais devem ser organizados para atender diversos usuários de forma dinâmica, conforme a necessidade dos consumidores.

- Elasticidade rápida: os recursos devem estar disponíveis de forma rápida e elástica, caso a demanda do usuário cresça em casos de pico de acessos, por exemplo.

- Serviços mensuráveis: os sistemas devem ter a capacidade de controlar e otimizar os recursos de forma automatizada e deve dispor de mecanismos capazes de medir a utilização desses recursos.

O NIST (2011) ainda apresenta os três modelos de serviços da computação em nuvem: software como serviço (SaaS - Software as a Service), plataforma como serviço (PaaS Platform as a Service) e infraestrutura como serviço (IaaS - Infrastructure as a Service), conforme visto a seguir:

- Software as a Service: são as aplicações que estão alocadas e sendo executadas na nuvem. É o serviço mais utilizado pelo consumidor final da $\mathrm{CN}$, ou seja, o usuário 
comum, tanto dos órgãos públicos e corporações, quanto os usuários avulsos da web.

- Platform as a Service: o usuário gerencia as aplicações alocadas na nuvem, porém não controla a infraestrutura. É um serviço mais utilizado pelos desenvolvedores das aplicações.

- Infrastructure as a Service: o usuário controla o armazenamento, sistemas e demais aplicações. Comumente, é mais utilizado pelas organizações, já que a capacidade de gerenciamento é maior.

Já os conceitos de modelos de implantação, também propostos por NIST (2011), são os seguintes: nuvem privada (private cloud), nuvem pública (public cloud), nuvem comunitária (community cloud) e nuvem híbrida (hybrid cloud).

- Nuvem privada: a estrutura da nuvem é dedicada exclusivamente à uma organização e seus stakeholders internos.

- Nuvem pública: o uso é aberto para o público em geral e atende diversos consumidores.

- Nuvem comunitária: a estrutura da nuvem é dedicada a um grupo específico, geralmente com um objetivo em comum.

- Nuvem híbrida: é composta por elementos de dois ou mais modelos que foram citados anteriormente.

Complementando os conceitos descritos acima, Armbrust et al. (2010) descrevem a nuvem privada como sendo grandes datacenters internos de organizações, que não estão disponíveis para o público em geral e descrevem nuvem pública como um modelo que possibilita o pay-as-you-go, um modelo que chama a atenção pelo seu sistema de pagamento por demanda de uso efetivo do serviço.

\subsection{Vantagens e desvantagens}

A grande discussão acerca da computação em nuvem se sustenta nas vantagens e desvantagens de sua utilização. Assim como toda tecnologia, produto ou serviço recente, ainda sofre com a resistência das pessoas e, consequentemente, das organizações, que analisam as vantagens e desvantagens do produto ou serviço para avaliar a viabilidade de sua utilização. A Computação em Nuvem traz várias vantagens para os seus usuários, em todos os níveis, do desenvolvedor que utiliza a plataforma, ao usuário que utiliza os softwares e às empresas que gerenciam seus dados.

As principais vantagens na utilização desta tecnologia estão baseadas na redução dos investimentos em infraestrutura de TI, bem como a diminuição de custos com pessoal 
especializado na área, o que torna o serviço financeiramente acessível, já que não necessita do planejamento e execução de projetos de infraestrutura interna de datacenters nas organizações, o que diminui bastante o custo com esse tipo de operação, por exemplo. Armbrust et al. (2010) citam alguns obstáculos e oportunidades para o crescimento da utilização da Computação em Nuvem, como por exemplo, a escalabilidade do armazenamento, o que possibilita o usuário de utilizar mais ou menos espaço de armazenamento de acordo com sua necessidade e de forma instantânea, o que pode gerar um ganho em escala muito grande e vantagem competitiva, já que não se faz necessária a elaboração de um projeto para montar uma estrutura local para aumentar a capacidade de armazenamento, o que tem como consequência, além da redução de custos, o tempo economizado. Armbrust et al. (2010) três aspectos na Computação em Nuvem que são vantajosos para os usuários:

- A impressão de recursos infinitos e a possibilidade de utilizar esses recursos sob demanda, o que elimina a necessidade alta de planejamento;

- Aumento nos recursos de Hardware somente quando necessário;

- Capacidade de pagar pelo uso no curto prazo.

Apesar das várias vantagens, o serviço também tem seus pontos negativos, ou pontos a melhorar. Depende da perspectiva do usuário ou da organização que utiliza a Computação em Nuvem. A questão da segurança dos dados e informações, apesar de ter evoluído muito nos últimos anos, ainda é vista por muitos usuários e organizações como um impeditivo para a utilização da tecnologia nos governos e grandes corporações, justamente pelo grau de confidencialidade e importância que os dados dessas organizações possuem.

No relatório do Tribunal de Contas da União (2014), uma das vantagens citadas foi o aumento da agilidade da Administração Pública Federal em acompanhar a evolução tecnológica, já que os processos formais de contratação pública é um fator que dificulta a manutenção de uma infraestrutura de TI, o que impede que existam atualizações que tornem o acompanhamento da evolução tecnológica possível. Além disso, também foi citado o suporte à iniciativas como o Big Data, outra tendência tecnológica em ascensão, e Dados Abertos, facilitando a transparência e maior participação da sociedade. Ainda de acordo com o Tribunal de Contas da União (2014),

uma política de governo que regulamente e também incentive o uso da computação em nuvem pode contribuir para o alcance de benefícios não só para a administração pública, mas também para organizações da iniciativa privada, sejam elas usuárias ou provedoras de recursos de tecnologia da informação. 
Quanto às vantagens de custo, as organizações, sejam elas públicas ou privadas, tem ganhos em escala muito grandes, já que uma estrutura tradicional de datacenter demanda diversos custos que envolvem um espaço adequado, custos altos com energia elétrica, manutenção dos equipamentos e profissionais. Já com a utilização dos serviços na nuvem, os custos diminuem consideravelmente, levando em conta que o serviço é pago de acordo com o uso, eliminando diversas despesas de uma estrutura tradicional de armazenamento e processamento de dados, aumentando a eficiência econômico-financeira das organizações. Ainda de acordo com Armsbrust et al. (2010), pay-as-you-go "capta mais diretamente o benefício econômico para o comprador", já que é o termo que define o principal método de pagamento e ainda citam o benefício econômico da elasticidade.

\subsection{Políticas públicas sobre o uso da CN}

Medeiros (2014), defendeu a necessidade de se ter políticas públicas mais consistentes na área de TI, no governo federal, dando especial atenção à computação em nuvem, como um item que deveria ser elevado à agenda de decisão de políticas públicas, se tornando uma ferramenta a ser utilizada em todos as esferas de governo.

O estudo realizado identificou que a maioria das normas, leis ou políticas públicas da área de TI estão dispersas, com sua gestão descentralizada em vários órgãos do governo federal, o que gera uma fragmentação da TI no governo, e aumenta a dificuldade de atuação dos gestores de TI.

Até então não foi identificada nenhuma política pública específica sobre computação em nuvem, embora exista um projeto de lei no legislativo federal sobre o tema. Em síntese, o que se identificou foi a possibilidade se ter a computação em nuvem como solucionadora de diversos problemas existentes no serviço público, mas ainda não se evidenciou um consenso entre os grupos de interesse, para que se avancem as discussões sobre o tema.

Portanto, apesar de o tema estar em evidência, ainda não existe nenhuma iniciativa específica para o uso da computação em nuvem, apesar de terem sido abordados assuntos diretamente ligados à esta tecnologia e a viabilidade de sua utilização, como a Estratégia de Governança Digital - EGD, o Plano Nacional de Banda Larga e o Marco Civil da Internet.

A Estratégia de Governança Digital, projeto implementado pelo Governo Federal no início de 2016, através do decreto $n^{\circ}$ 8.638. A utilização da Computação em Nuvem é citada no 
documento como uma das iniciativas de um dos objetivos estratégicos definidos, o OE.07, descrito a seguir:

Os dados, os processos, os sistemas de informação, os serviços e a infraestrutura tecnológica devem ser compartilhados entre os órgãos e entidades de forma a reduzir custos e desperdícios e evitar esforços desnecessários e perda de dados e informações (Presidência da República, 2016).

A Iniciativa Estratégica IE.07.02 visa "compartilhar estruturas de datacenter nos órgãos e ampliar a oferta de serviços em nuvem nas empresas de governo". (Presidência da República, 2016). A Iniciativa é apenas parte de um projeto que, apesar de ser bastante genérico, é mais um passo importante para a utilização da $\mathrm{CN}$ nos órgãos públicos e para posteriores políticas mais específicas.

O Plano Nacional de Banda Larga é um projeto que visava popularizar a utilização da Internet no país, disponibilizando o serviço em lugares remotos, além de melhorar a qualidade de conexão, fato de fundamental importância para a implantação da computação em nuvem, tendo em vista que Internet de qualidade é um dos requisitos necessários à utilização da $\mathrm{CN}$. Apesar de não ter alcançado todos os objetivos propostos inicialmente através do decreto $\mathrm{n}^{\circ}$ 7.175, de 12 de maio de 2010, o governo já prepara uma atualização para dar continuidade ao plano e tem projeto previsto ainda para o ano de 2016.

O Marco Civil da Internet (Lei 12.965/2014) pode ser considerado uma constituição para o ambiente virtual e afeta diretamente o uso da Computação em Nuvem, principalmente no que tange à privacidade dos dados. A utilização de datacenters acabou não sendo incluída no documento final, não havendo assim uma legislação específica para a utilização dos centros de dados.

Essas iniciativas acabam impulsionando novos projetos, propostas e discussões, como o PL 5276/2016, que “dispõe sobre o tratamento de dados pessoais para a garantia do livre desenvolvimento da personalidade e da dignidade da pessoa natural". O incentivo à essas propostas, mesmo ainda não sendo específicas para a $\mathrm{CN}$, é benéfico, já que o Brasil ainda é muito carente de políticas públicas na área de Tecnologia da Informação, o que nos deixa atrasados em relação à diversos países que avançam nesse sentido. Segundo Medeiros (2014), é necessário pensar em políticas públicas para a computação em nuvem, como alternativa para minimizar o gap que existe entre a evolução tecnológica e sua incorporação nas atividades do Estado. 
Para facilitar esse processo de inserção de tecnologias e criação de políticas públicas, é necessário que profissionais de Tecnologia da Informação tecnicamente capacitados assumam posições no serviço público, fomentando a discussão e introduzindo a utilização dessas tecnologias nas organizações públicas, o que possibilitaria o avanço do Estado. Ainda assim, Medeiros e Sousa Neto (2016) afirmam que a adoção de novas tecnologias na esfera pública se torna mais complexa em função de algumas características burocráticas do serviço público.

O Tribunal de Contas da União, através da Secretaria de Fiscalização de Tecnologia da Informação - SEFTI, fez um estudo para avaliar os riscos em contratações de serviços de nuvem na Administração Pública Federal - APF. O resultado foi o acórdão 1.739/2015-TCU-Plenário, que contém diversas informações sobre o serviço, com o objetivo de facilitar as futuras auditorias do Tribunal de Contas da União em contratações de serviços de nuvem. Como consequência deste estudo, através da Secretaria de Tecnologia da Informação, o Ministério do Planejamento, Orçamento e Gestão elaborou um documento com recomendações sobre a contratação de Serviços de Computação em Nuvem por órgãos do Governo Federal. O documento orienta os gestores desses órgãos a contratar os serviços, evidenciando as vantagens, principalmente de custos, que a CN proporciona. Este é um importante passo, pois direciona e dá subsídios para que os gestores dos órgãos da APF utilizem o serviço, observando as vantagens oferecidas, otimizando e tornando mais eficiente a utilização dos recursos de TI.

A princípio, o governo não irá alocar todos os seus dados na nuvem, já que muitas informações confidenciais e estratégicas devem ser mantidas sob os cuidados do Estado. Porém, fazendo menção ao princípio da transparência, as informações que devem ser compartilhadas com a sociedade devem estar alocadas na nuvem. No artigo $1^{\circ}$, do decreto $\mathrm{n}^{\circ} 8.135$, de 4 de novembro de 2014, que dispõe sobre as comunicações de dados na APF, podemos observar a posição do governo quanto ao tema:

As comunicações de dados da administração pública federal direta, autárquica e fundacional deverão ser realizadas por redes de telecomunicações e serviços de tecnologia da informação fornecidos por órgãos ou entidades da administração pública federal, incluindo empresas públicas e sociedades de economia mista da União e suas subsidiárias (Presidência da República, 2013)

A utilização de recursos no serviço público se torna mais dependente de eficiência a cada dia que passa, tendo em vista a escassez desses recursos, principalmente financeiros e materiais exigindo que os servidores utilizem de forma mais eficiente possível aquilo que estiver à sua disposição. No caso da computação em nuvem, fica evidente o melhoramento e a eficiência de 
utilização dos recursos, já que se trata de um serviço completamente flexível em diversos aspectos, como visto anteriormente nas vantagens apresentadas, o que torna a opção ainda mais vantajosa para as organizações públicas. Ainda de acordo com Medeiros e Sousa Neto (2016), a atuação dos agentes públicos deve caminhar cada vez mais próxima dos pilares da transparência, responsabilidade nos gastos públicos e eficiência, o que pode ser proporcionado pela CN na área de TI.

\section{Metodologia}

O presente capítulo de metodologia consiste na apresentação de conceituações de caracterização da pesquisa, coleta e análise de dados, assim como tais foram aplicados. Sendo resumidos na Tabela 1 , em seguida:

\section{Tabela 1}

Síntese da Metodologia

Caracterização da Pesquisa

\begin{tabular}{l|c|c}
\hline Natureza das Variáveis & Qualitativa & Quantitativa \\
\hline Objetivo e Grau do Problema & Exploratória & Descritiva \\
\hline Amplitude e Profundidade & \multicolumn{2}{|c}{ Estudo de Campo } \\
\hline
\end{tabular}

Coleta de Dados

\begin{tabular}{c|c|c}
\hline \multicolumn{2}{c|}{ Qualitativos } & Quantitativos \\
\hline Literatura de & $\begin{array}{c}\text { Experiência dos } \\
\text { Pesquisadores }\end{array}$ & Questionário Fechado \\
\cline { 2 - 3 } Computação em Nuvem & Escala de Tipo Likert \\
\hline
\end{tabular}

Análise dos Dados

\begin{tabular}{c|c}
\hline Confiabilidade de Consistência Interna & Coeficiente Alfa de Cronbach $(\alpha)$ \\
\hline Interpretativista & Análise e Discussão dos Resultados \\
\hline
\end{tabular}

Fonte: Pesquisa (2018)

Sobre a caracterização da pesquisa, o trabalho assume uma postura exploratória quanto aos seus objetivos e ao grau do problema ao formular e definir as variáveis qualitativamente (Vergara, 1998) onde não foram empregadas relações entre variáveis, mas, sim, a descoberta destas, além da caracterização da abordagem mediante os conceitos da literatura e experiência dos pesquisadores (Köche, 2011). 
Contudo, na análise dos dados, foram empregadas relações entre as variáveis com base no cálculo do Coeficiente de Alfa de Cronbach $(\alpha)$ responsável por mensurar a Consistência Interna dos instrumentos de coleta de dados, sendo este o enfoque essencialmente descritivo de natureza quantitativa de acordo com a equação:

$$
\propto=\frac{k}{k-1}\left(1-\frac{\sum_{i=1}^{k} \partial^{2} i}{\partial_{\text {soma }}^{2}}\right)
$$

para $k$ representa a quantidade de variáveis, respondidas por $n$ entrevistados para a matriz $x_{i j}$ formada pelo escore $i(i=1,2, \ldots, k)$ e respondente $j(j=1,2, \ldots, n)$, onde $\partial^{2}{ }_{\text {soma }}$ representa a variância total dos respondentes e $\partial^{2}$ são calculadas mediante:

$$
\partial^{2}=\frac{\sum(x-\bar{x})^{2}}{n}
$$

Inicialmente com o escore englobando todas as variáveis seguido pela análise individual dos grupos, e seus respectivos modelos otimizados (com a retirada de variáveis menos ou não relacionadas à categoria), que compõem o questionário. Os dados foram tratados com auxílio do Software IBM SPSS $®$.

Além do cálculo do Coeficiente de Alfa de Cronbach, existem outros dois métodos de verificação de Confiabilidade de um instrumento de coleta de dados, o teste/reteste, quando o questionário é aplicado mais de uma vez e os resultados são comparados, e o Split-half, ou divisão em metades, onde a amostra é dividida em duas partes e realizada uma comparação através do Coeficiente de Correlação Linear de Pearson (Corrente, 2009; Hair, Babin, Money, $\&$ Samouel, 2005).

A Tabela 2, em seguida, reúne o posicionamento de três obras, que apesar de se contraporem em relação a exatidão, seguem a mesma linha de tendência nas classificações progressivas dos valores. 
Tabela 2

Regras Práticas sobre a Dimensão do Coeficiente Alfa de Cronbach

\begin{tabular}{|c|c|c|c|c|c|}
\hline \multicolumn{2}{|c|}{ Landis e Koch (1977) } & \multicolumn{2}{|c|}{ George e Mallery (2003) } & \multicolumn{2}{|c|}{$\begin{array}{l}\text { Hair, Babin, Money, \& Samouel } \\
\text { (2005) }\end{array}$} \\
\hline Valor de Alfa & $\begin{array}{l}\text { Consistência } \\
\text { Interna }\end{array}$ & Valor de Alfa & $\begin{array}{l}\text { Consistência } \\
\text { Interna }\end{array}$ & Valor de Alfa & $\begin{array}{l}\text { Consistência } \\
\text { Interna }\end{array}$ \\
\hline$<0,21$ & Pequeno & $<0,51$ & Inaceitável & $<0,6$ & Baixa \\
\hline $0,21<0,4$ & Razoável & $0,51<0,6$ & Pobre & $0,6<0,7$ & Moderada \\
\hline $0,41<0,6$ & Moderado & $0,61<0,7$ & Questionável & $0,7<0,8$ & Boa \\
\hline $0,61<0,8$ & Substancial & $0,71<0,8$ & Aceitável & $0,8<0,9$ & Muito Boa \\
\hline \multirow{2}{*}{$0,8<$} & \multirow{2}{*}{ Quase Perfeito } & $0,81<0,9$ & Bom & \multirow{2}{*}{$0,9<$} & \multirow{2}{*}{ Excelente } \\
\hline & & $0,91<$ & Excelente & & \\
\hline
\end{tabular}

Fonte: Pesquisa (2017)

Para análise das categorias constantes no desenvolvimento (Resultados e Discussões) do presente trabalho foram escolhidos os critérios adotados por Hair et al. (2005), já que são autores de maior relevância da área e adotados com maior frequência nos trabalhos acadêmicocientíficos. Entretanto, no decorrer da apresentação dos resultados Landis e Koch (1977) e George e Mallery (2003) serão citados de maneira complementar.

\section{Resultados e discussões do trabalho}

O presente trabalho está baseado em três pilares, o primeiro deles é contato e a vivência com pesquisadores de renome na área, além disso, no segundo pilar, muitos documentos de cunho técnico e normativo (Regulamentos do Tribunal de Contas da União e Definições do NIST) serviram de base para a construção das variáveis e divisão das categorias, por fim, o terceiro pilar consiste no embasamento da literatura, tanto pesquisas internacionais, como Armsbrust et al. (2010), Vaquero et al. (2009) e Marston et al., 2010, quanto nacionais, Medeiros (2014) e Veras (2012).

\section{Tabela 3}

Confiabilidade de Coerência Interna do Instrumento de Coleta de Dados

\begin{tabular}{c|c}
\hline Alfa de Cronbach & N de itens \\
\hline, 867 & 46 \\
\hline
\end{tabular}

Fonte: Pesquisa (2018) 
Em primeira instância, vale a pena salientar que, em relação ao instrumento analisado de conjunto (com a presença de todas as categorias), o Coeficiente de Alfa de Cronbach obteve um escore de 0,867, considerado quase perfeito por Landis e Koch (1977), bom por George e Mallery (2003) e muito bom por Hair et al. (2005). Sendo assim, nota-se uma unanimidade na literatura que confirmam o confiança do instrumento de coleta de dados analisado no presente trabalho.

\subsection{Categoria capacitação}

A primeira categoria, responsável por questões de Capacitação, incialmente, possui quatro variáveis, contudo ao verificar a confiabilidade o escore resultante apresentou negatividade, -0,170. Hair et al. (2005, p. 200) comentam que esse fator pode ser oriundo de uma redação negativa, assim como pode ser observado na Tabela 4 em x29, com a palavra "não" e x45, com a palavra "limitações".

\section{Tabela 4}

Composição da Categoria Capacitação

\begin{tabular}{c|l|c|c}
\hline \multicolumn{1}{c|}{ Variáveis } & Alfa de Cronbach & $\begin{array}{c}\text { Confiabilidade } \\
\text { Otimizada }\end{array}$ \\
\cline { 3 - 3 }$\times 16$ & $\begin{array}{l}\text { A computação em nuvem já está incorporada à vida das } \\
\text { pessoas, este fator influencia a decisão do governo em } \\
\text { utilizá-la. }\end{array}$ & $\mathrm{X}$ & $\mathrm{X}$ \\
\hline $\mathrm{x} 27$ & $\begin{array}{l}\text { Os profissionais da área de TI do governo já estão } \\
\text { capacitados para a computação em nuvem }\end{array}$ & $\mathrm{X}$ & $\mathrm{X}$ \\
\hline $\mathrm{x} 29$ & $\begin{array}{l}\text { Os servidores públicos não possuem conhecimento } \\
\text { suficiente sobre computação em nuvem. }\end{array}$ & $\mathrm{X}$ & $\mathrm{X}$ \\
\hline $\mathrm{x} 45$ & $\begin{array}{l}\text { Existem limitações técnicas para o uso da computação em } \\
\text { nuvem. }\end{array}$ & \\
\hline
\end{tabular}

Fonte: Pesquisa (2018)

Com a remoção destas duas variáveis o valor do Alfa de Cronbach saiu de valor negativo ao classificado por Landis e Koch (1977) como moderado, em contraste com Hair et al. (2005) e George e Mallery (2003), que classificam como baixo e pobre, respectivamente. Embora Hair et al. (2005) comentem que "coeficientes mais baixos [inferior a 0,7] possam ser aceitáveis, dependendo dos objetivos da pesquisa", tal como o presente trabalho que visa criar um modelo de referência exploratório para que outros métodos possam surgir a partir deste. 


\subsection{Categoria implantação}

Desde a primeira mensuração, a categoria Implantação alçou resultados satisfatórios, onde as três variáveis do modelo possuem um valor de alfa considerado aceitável por George e Mallery (2003), totalizando 0,784, assim como representado na Tabela 5.

\section{Tabela 5}

Composição da Categoria Implantação

\begin{tabular}{|c|c|c|c|}
\hline \multicolumn{2}{|r|}{ Variáveis } & Alfa de Cronbach & $\begin{array}{l}\text { Confiabilidade } \\
\text { Otimizada }\end{array}$ \\
\hline $\mathrm{x} 31$ & $\begin{array}{l}\text { Já existe alguma aplicação de computação em nuvem onde } \\
\text { trabalho. }\end{array}$ & $\mathrm{X}$ & \\
\hline $\mathrm{x} 36$ & $\begin{array}{l}\text { Um órgão do governo está desenvolvendo aplicações de } \\
\text { computação em nuvem. }\end{array}$ & $\mathrm{X}$ & $\mathrm{X}$ \\
\hline x37 & $\begin{array}{l}\text { Um órgão do governo já adquiriu uma aplicação baseada em } \\
\text { computação em nuvem. }\end{array}$ & $X$ & $X$ \\
\hline
\end{tabular}

Fonte: Pesquisa (2018)

Com a remoção da variável menos correlacionada com as outras duas, o índice pouco variou positivamente (subindo para 0,800 ), fato que demonstra a necessidade de manter as três variáveis no construto, ao invés de excluir a com menos afinidade, já que o grupo se mantem consistente com a presença de tal.

\subsection{Categoria infraestrutura}

Com a presença de cinco variáveis na categoria Infraestrutura (ver Tabela 6), Landis e Koch (1977) classificam o resultado do Coeficiente de Alfa de Cronbach de apenas 0,133 como pequeno, pensamento unânime aos demais autores. 
Tabela 6

Composição da Categoria Infraestrutura

\begin{tabular}{|c|c|c|c|}
\hline \multicolumn{2}{|r|}{ Variáveis } & Alfa de Cronbach & $\begin{array}{l}\text { Confiabilidade } \\
\text { Otimizada }\end{array}$ \\
\hline & &, 133 & ,712 \\
\hline $\mathrm{x} 14$ & $\begin{array}{l}\text { O governo não consegue acompanhar a velocidade com que } \\
\text { as mudanças tecnológicas acontecem. }\end{array}$ & $\mathrm{X}$ & $\mathrm{X}$ \\
\hline $\mathrm{x} 15$ & $\begin{array}{l}\text { Ainda falta muito para o governo utilizar computação em } \\
\text { nuvem. }\end{array}$ & $\mathrm{X}$ & $\mathrm{X}$ \\
\hline $\mathrm{x} 34$ & $\begin{array}{l}\text { Existem estudos sobre a utilização da computação em nuvem } \\
\text { no governo. }\end{array}$ & $\mathrm{X}$ & \\
\hline $\mathrm{x} 8$ & Internet Banda Larga é prioritária no Brasil. & $X$ & \\
\hline $\mathrm{x} 9$ & $\begin{array}{l}\text { Internet Banda Larga é um entrave para a computação em } \\
\text { nuvem. }\end{array}$ & $\mathrm{X}$ & \\
\hline
\end{tabular}

Fonte: Pesquisa (2018)

Entretanto, com a permanência de x14 e x15 e a remoção das restantes, esse valor subiu para 0,712 , considerado por Hair et al. $(2005$, p. 200) como o mínimo confiável por grande parte dos pesquisadores.

\subsection{Categoria modelo}

Ao ser calculado o valor de Alfa de Cronbach para as três variáveis do construto Modelo, índices baixos foram resultantes, insuficientes para demonstrar a confiabilidade deste (Tabela 7).

\section{Tabela 7}

Composição da Categoria Modelo

\begin{tabular}{|c|c|c|c|}
\hline \multirow{2}{*}{\multicolumn{2}{|c|}{ Variáveis }} & Alfa de Cronbach & $\begin{array}{l}\text { Confiabilidade } \\
\text { Otimizada }\end{array}$ \\
\hline & & ,199 & ,683 \\
\hline $\mathrm{x} 40$ & O governo deve utilizar o modelo de nuvem privada. & $\mathrm{X}$ & $\mathrm{X}$ \\
\hline $\mathrm{x} 41$ & $\begin{array}{l}\text { O modelo de nuvem pública é o mais adequado para o } \\
\text { governo. }\end{array}$ & $X$ & $\mathrm{X}$ \\
\hline $\mathrm{x} 42$ & $\begin{array}{l}\text { O governo deve pensar em um modelo híbrido de computação } \\
\text { em nuvem. }\end{array}$ & $\mathrm{X}$ & \\
\hline
\end{tabular}

Fonte: Pesquisa (2018) 
Sendo assim, apenas com a permanência das variáveis x40 e x41, a confiabilidade passou a 0,683 (valor moderado), muito próximo a classificação de bom pelos critérios de Hair et al. (2005).

\subsection{Categoria organizacional}

Inicialmente, devido a uma covariância média negativa entre itens da Categoria Organizacional, o Coeficiente de Alfa de Cronbach também mediu índices negativos $(-, 874)$, assim como registrado na Tabela 8. O estudo para encontrar novas variáveis pode trazer algum nível de confiança ao modelo.

\section{Tabela 8}

Composição da Categoria Organizacional

\begin{tabular}{|c|c|c|c|}
\hline \multicolumn{2}{|r|}{ Variáveis } & Alfa de Cronbach & $\begin{array}{l}\text { Confiabilidade } \\
\text { Otimizada }\end{array}$ \\
\hline $\mathrm{x} 13$ & $\begin{array}{l}\text { Os gestores de TI possuem resistência em utilizar a } \\
\text { computação em nuvem. }\end{array}$ & $X$ & \\
\hline $\mathrm{x} 19$ & $\begin{array}{l}\text { Computação em nuvem tem um forte apelo para a maior } \\
\text { eficiência dos processos. }\end{array}$ & $X$ & $\mathrm{X}$ \\
\hline $\mathrm{x} 3$ & $\begin{array}{l}\text { O governo necessita da computação em nuvem para melhorar } \\
\text { seus processos. }\end{array}$ & $X$ & $X$ \\
\hline
\end{tabular}

Fonte: Pesquisa (2018)

A remoção da variável x13 aumentou consideravelmente a confiança do Componente Organizacional, saindo de valor negativo para o valor positivo de 0,300 , entretanto o coeficiente continua com valor bem abaixo do recomendado por Hair et al. (2005).

\subsection{Categoria políticas}

Dentre todos os componentes do presente trabalho de pesquisa, os índices de Coeficiente de Alfa de Cronbach mais expressivos são da Categoria Políticas, totalizando 0,823 (Tabela 9) com a presença de vinte variáveis. 
Tabela 9

Composição da Categoria Políticas

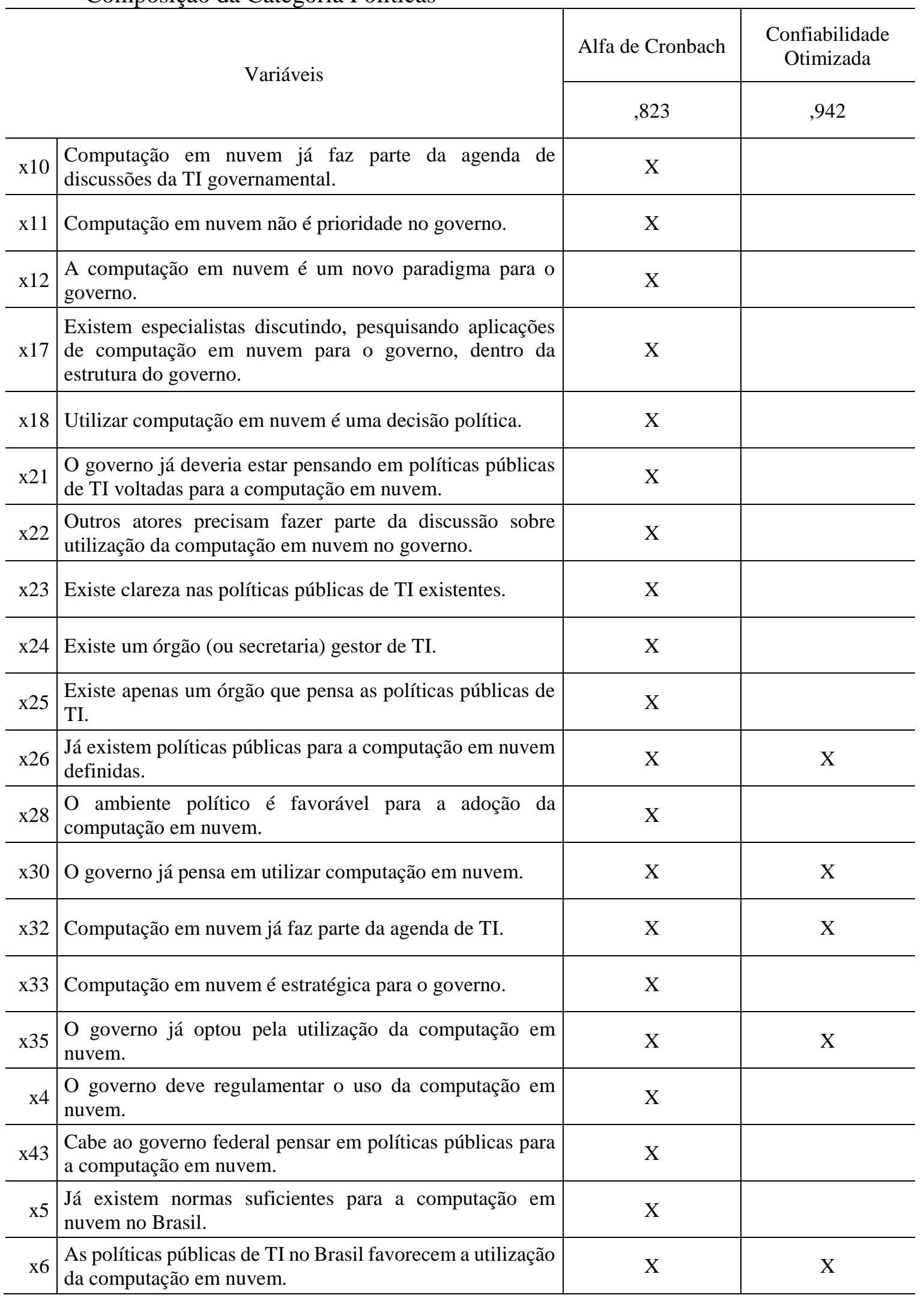

Fonte: Pesquisa (2018) 
Apesar de possuir confiabilidade de nível muito bom (Tabela 9), com a retirada das variáveis menos correlatas, a categoria de Políticas consegue alcançar escores próximos a 0,950 onde Hair et al. (2005) alertam que os itens devem passar por inspeção que objetivem detectar se os mesmos aspectos do conceito estão sendo mensurados, nos resultados com as cinco variáveis mais correlatas o Alfa chegou a 0,942.

Os resultados dessa categoria são excelentes, sendo assim as atuais variáveis tem capacidade potencial de se tornarem latentes, proposta cabível de desenvolvimento em estudos posteriores resultantes de possíveis recategorizações, principalmente subcategorizações, deste construto.

\subsection{Categoria recursos}

A Categoria Recursos, representada por seis variáveis alcançou níveis moderados de Alfa de Cronbach $(0,605)$, tal qual demonstrado na Tabela 10.

\section{Tabela 10}

Composição da Categoria Recursos

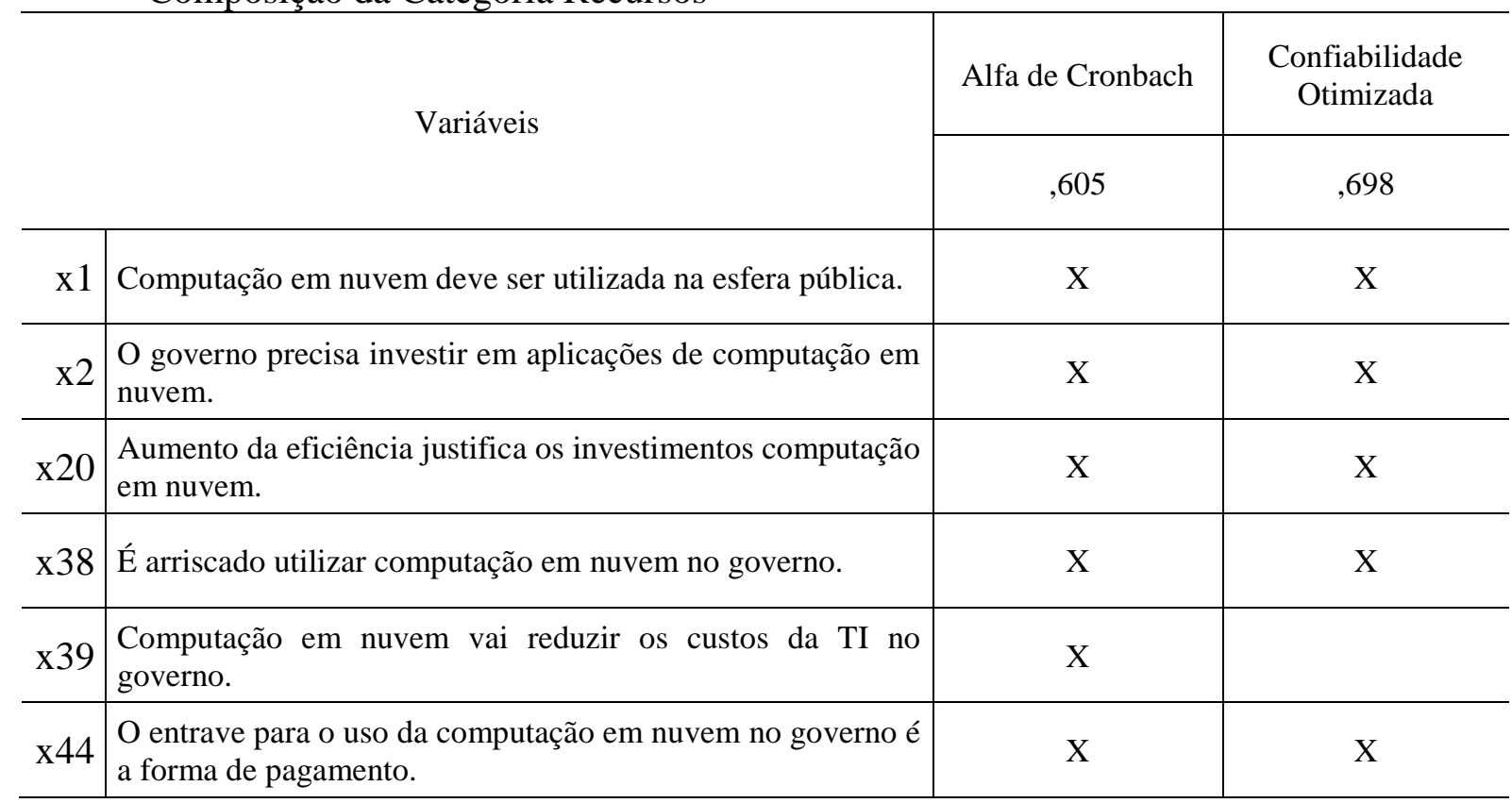

Fonte: Pesquisa (2018)

Com a retirada da variável x39 (Computação em nuvem vai reduzir os custos da TI no governo) o Coeficiente de Alda de Cronbach foi para 0,698, conseguindo intensidade de associação moderada, com proximidade a um nível bom nos conformes mencionados por Hair 
et al. (2005), sendo esse o escore de confiabilidade máximo alcançado pelo modelo, caso outra variável seja removida sua significância começa e ser reduzida.

\subsection{Categoria segurança}

Os resultados de Confiabilidade para a categoria Segurança (Tabela 11) foram abaixo dos aceitáveis recomendados pela literatura e é composto apenas por duas variáveis que apresentam baixa correlação, a adição de novas variáveis pode aumentar a importância desse componente.

\section{Tabela 11}

Composição da Categoria Segurança

\begin{tabular}{r|l|c}
\hline \multicolumn{1}{c|}{ Variáveis } & \multirow{2}{*}{ Alfa de Cronbach } \\
\hline x46 & Computação em nuvem traz mais segurança para o governo. & \\
\hline$x 7$ & $\begin{array}{l}\text { Segurança da informação é um fator de risco para a computação em nuvem no } \\
\text { serviço público. }\end{array}$ &, 344 \\
\hline
\end{tabular}

Fonte: Pesquisa (2018)

Sendo assim, nos termos apresentados pelos autores, o escore apresentado seria classificado como razoável por Landis e Koch (1977) e inaceitável e baixo por George e Mallery (2003) e Hair et al. (2005), respectivamente.

\section{Conclusões}

Embora os resultados preliminares apontem valores baixos para alguns construtos, outros, como Políticas e Implantação, apresentam resultados significantes para a validação do modelo no que concerne os objetivos do presente trabalho, a confiabilidade do instrumento de coleta de dados, como sintetizado a seguir na Tabela 12. 
Tabela 12

Síntese dos Resultados

\begin{tabular}{|c|c|c|c|c|c|c|}
\hline \multirow{2}{*}{ Categorias } & \multicolumn{2}{|c|}{$\begin{array}{l}\text { Coeficiente de } \\
\text { Confiabilidade }\end{array}$} & \multicolumn{2}{|c|}{ Quantidade de Variáveis } & \multicolumn{2}{|c|}{ Consistência Interna } \\
\hline & Inicial & Final & Inicial & Final & Inicial & Final \\
\hline Capacitação &,- 170 & ,599 & 4 & 2 & Baixa & Baixa \\
\hline Implantação & ,784 &, 800 & 3 & 2 & Boa & Muito Boa \\
\hline Infraestrutura &, 133 & ,712 & 5 & 2 & Baixa & Boa \\
\hline Modelo & , 199 & 683 & 3 & 2 & Baixa & Moderada \\
\hline Organizacional &,- 874 & ,300 & 3 & 2 & Baixa & Baixa \\
\hline Políticas & ,823 & ,942 & 20 & 5 & Muito Boa & Excelente \\
\hline Recursos & 605 & 698 & 6 & 5 & Moderada & Moderada \\
\hline Segurança & ,344 & ,344 & 2 & 2 & Baixa & Baixa \\
\hline
\end{tabular}

Fonte: Pesquisa (2017)

* De acordo com os critérios de Hair et al. (2005)

Sendo assim, com base na literatura, de maneira preliminar, pode-se afirmar que o instrumento tal qual aplicado obteve apenas grau de confiabilidade em três construtos: Recursos (Nível Moderado), Implantação (Nível Bom) e Políticas (Muito Bom). No entanto, com o auxílio do Software IBM $®$ SPSS $®$ foram obtidos resultados otimizados do questionário ao serem removidas variáveis com correlação baixa ou oposta ao agrupamento. Os resultados foram significantes para os demais construtos, com exceção de Segurança, por possuir apenas duas variáveis, não sofreu alteração.

A primeira recomendação consiste em uma validação com amostras maiores e a internacionalização da pesquisa, bem como a abordagem de novos aspectos em relação à computação em nuvem. Ainda assim, uma das maiores contribuições dos resultados apresentados é a possibilidade de modelagem de Políticas Públicas de Computação em Nuvem em Equações Estruturais. Com base na literatura de tecnologia da informação, ficam eminentes as possibilidades de expansão dos construtos apresentados, com a inclusão de diversas variáveis que possam ampliar o modelo com maior completude. Além disso, existem possibilidades que 
os construtos desta pesquisa possam crescer ao ponto de se tornarem independentes, avaliando questões mais específicas das políticas públicas de computação em nuvem.

\section{Referências}

Armbrust, M., Fox, A., Griffith, R., Joseph, A. D., Katz, R., Konwinski, A., \& Zaharia, M. (2010). A view of cloud computing. Communications of the ACM, 53(4), 50-58.

Câmara dos Deputados. (2016). Projeto de Lei no 5276/2016, de 13 de maio de 2016. Dispõe sobre o tratamento de dados pessoais para a garantia do livre desenvolvimento da personalidade e da dignidade da pessoa natural. Pl 5276/2016. Brasília, DF. Recuperado em $30 \quad$ outubro, 2016, http://www.camara.gov.br/proposicoesWeb/fichadetramitacao?idProposicao=2084378

Corrente, José Eduardo. (2009). Medidas de Confiabilidade. In Vieira, Sônia. Como Elaborar Questionários. São Paulo: Atlas.

Flick, Uwe (2009). Qualidade na pesquisa qualitativa: Coleção Pesquisa Qualitativa. Bookman.

George, D., \& Mallery, P. (2003). SPSS for Windows step by step: A simple guide and reference. 11.0 update (4a ed.). Boston: Allyn \& Bacon.

Gibbs, Graham. (2009). Análise de dados qualitativos: coleção pesquisa qualitativa. Bookman. Gil, Antônio Carlos (2008). Métodos e Técnicas de Pesquisa Social (6a ed.) São Paulo: Atlas.

Guerra, Isabel Carvalho (2006). Pesquisa qualitativa e análise de conteúdo: sentidos e formas de uso. Estoril: Principia, publicações universitárias e cientificas.

Hair Junior, J. F., Babin, B., Money, A. H., \& Samouel, P. (2005). Fundamentos de métodos de pesquisa em administração. Porto Alegre: Bookman.

Kim, W. (2009). Cloud computing: today and tomorrow. Journal of Object Technology,8 (1).

Kingdon, John W. (2014). Agendas, alternatives, and public policies. 2nd Pearson Education Limited.

Köche, J. C. (1997). Fundamentos de metodologia científica: teoria da ciência e prática da pesquisa. Petrópolis: Vozes.

Kundra, V. (2011). Federal Cloud Computing Strategy. Recuperado de http://www.whitehouse.gov/sites/default/files/omb/assets/egov_docs/vivek-kundrafederalcloud-computing-strategy-02142011.pdf

Landis, J. R., \& Koch, G. G. (1977). The measurement of observer agreement for categorical data. Biometrics, 159-174.

Lapponi, J. C. (2005). Estatística usando excel. Rio de Janeiro: Elsevier.

Marconi, M. D. A., \& Lakatos, E. M. (2003). Fundamentos de pesquisa metodológica científica (5a ed.). São Paulo: Atlas.

Marston, S., Li, Z., Bandyopadhyay, S., Zhang, J., \& Ghalsasi, A. (2011). Cloud computing: the business perspective. Decision Support Systems, 51 (1), 176-189.

Medeiros, M. F. M. (2014). Computação em nuvem no governo: caminhos para a formação de uma agenda governamental. (Tese de doutorado. Universidade Federal do Rio Grande do Norte, Natal, RN, Brasil). 
Medeiros, M. F. M. \& Sousa Neto, M. V. (2014). Cloud computing and Internet governance: trend topics in the Brazilian public service. Anais do 12th CONTECSI, São Paulo, SP, Brasil.

Medeiros, M. F. M. \& Sousa Neto, M. V. (2016). Uso da Computação em Nuvem no Setor Público: um Estudo de Caso com Gestores de TI do Estado do Rio Grande do Norte e do Governo Federal. Revista Gestão \& Tecnologia, 16 (1), 161-182.

Ministério do Planejamento, Orçamento e Gestão. (2016). Boas Práticas, Orientações e Vedações Para Contratação de Serviços de Computação em Nuvem. Brasília, DF, Recuperado de: http://www.governoeletronico.gov.br/eixos-de-atuacao/governo/sistemade-administracao-dos-recursos-de-tecnologia-da-informacao-sisp/ncti-nucleo-de-

contratacoes-de-tecnologia-da-informacao/orientacoes-e-vedacoes-para-contratacao-desolucoes-de-ti.

National Institute of Standards and Technology -NIST (2011). The NIST definition of cloud computing. Gaithersburg, MD: NIST.

Presidência da República (2010). Decreto no 7.175, de 12 de maio de 2010. Institui o Programa Nacional de Banda Larga - PNBL; dispõe sobre remanejamento de cargos em comissão; altera o Anexo II ao Decreto no 6.188, de 17 de agosto de 2007; altera e acresce dispositivos ao Decreto no 6.948, de 25 de agosto de 2009; e dá outras providências. Plano Nacional de Banda Larga. Brasília, DF. Recuperado em: http://www.planalto.gov.br/ccivil_03/_Ato2007-2010/2010/Decreto/D7175.htm.

Presidência da República. (2013). Decreto $\mathrm{n}^{\circ}$ 8.135, de 04 de novembro de 2013. Dispõe sobre as comunicações de dados da administração pública federal direta, autárquica e fundacional, e sobre a dispensa de licitação nas contratações que possam comprometer a segurança nacional. Decreto $N^{o}$ 8.135, de 4 de novembro de 2013. Brasília, DF. Recuperado em: http://www.planalto.gov.br/ccivil_03/_Ato2011-2014/2013/Decreto/D8135.htm

Presidência da República. (2016). Decreto nº 8.638, de 15 de janeiro de 2016. Institui a Política de Governança Digital no âmbito dos órgãos e das entidades da administração pública federal direta, autárquica e fundacional. Estratégia de Governança Digital. Recuperado em: http://www.planalto.gov.br/CCIVIL_03/_Ato2015-2018/2016/Decreto/D8638.htm

Rauen, C. V. (2011). Mudança tecnológica e definição de agenda de políticas públicas: regulação para universalização da banda larga no Brasil. Revista de Direito, Estado e Telecomunicações. v. 3, n. 1, p. 89-110.

Santos, U.; Amelotti, L. A.; Villar, F. (2012). Adoção de computação em nuvem e suas motivações. CSA.

Schramm, Wilbur (1971). Notes on Case Studies of Instructional Media Projects. Institute of Education Sciences. Stanford University - California Institute for Communication Research. Recuperado em: 〈eric.ed.gov〉. Acesso em: 10 abr. 2016.

Tribunal de Contas da União. (2015). Acórdão no 1739/2015, de 2015. Acórdão 1.739/2015tcu-plenário. Recuperado em: http://portal.tcu.gov.br/comunidades/fiscalizacao-detecnologia-da-informacao/atuacao/destaques/computacao-em-nuvem.htm

Tigre, P. B., \& Noronha, V. B. (2013). Do mainframe à nuvem: inovações, estrutura industrial e modelos de negócios nas tecnologias da informação e da comunicação. Revista de Administração, [s.1.], v. 48, n. 1, p.114-127. Trimestral. Business Department, School of Economics, Business \& Accounting USP. http://dx.doi.org/10.5700/rausp1077.

Triviños, A. N. S. (1987). Introdução à pesquisa em ciências sociais: a pesquisa qualitativa em educação. São Paulo: Atlas.

Vaquero, L. M., Rodero-Merino, L., Caceres, J., \& Lindner, M. (2008). A break in the clouds. ACM SIGCOMM Computer Communication Review, 39(1), 50-55. doi:10.1145/1496091.1496100 
Veras, Manoel.(2012). Cloud Computing: Nova Arquitetura da TI. São Paulo: Brasport.

Vieira, S. (2009). Como elaborar questionários. São Paulo: Atlas.

Vergara, S. C. (1998). Projetos e relatórios de pesquisa em administração. (2a ed.). São Paulo: Atlas.

Xu, X. (2012). From cloud computing to cloud manufacturing. Robotics and ComputerIntegrated Manufacturing. v. 28, p. 75-86.

Yin, R. K (2001). Estudo de Caso: Planejamento e Métodos (2a ed.). Porto Alegre: Bookman. 